\title{
AMÉRICA LATINA: UNA DEUDA PENDIENTE DE LA POLIITICA EXTERIOR DE INDIA
}

\author{
LATIN AMERICA: AN OUTSTANDING DEBT IN INDIA'S \\ FOREIGN POLICY
}

\author{
Paola Andrea Baroni ${ }^{1}$ \\ ORCID: 0000-0003-4553-4893
}

\section{RESUMEN}

El primer ministro Modi ha inyectado un nuevo dinamismo en la conducción de las relaciones exteriores de India, asumiendo un rol más influyente en los asuntos regionales e internacionales. Desde su asunción, ha viajado a casi todos los continentes, pero en América Latina solo ha estado en dos ocasiones en reuniones multilaterales. América Latina ofrece diversas oportunidades a India, en términos de comercio (seguridad alimentaria y energética), inversiones y en el ámbito de cooperación sur-sur. Sin embargo, India muestra una negligencia benigna hacia la región. En el presente artículo se analiza la política exterior del gobierno de Modi y, a la luz de esta misma, la relación con América Latina, buscando establecer aquellas oportunidades y obstáculos en la relación bilateral.

Palabras clave: Política exterior; oportunidades; obstáculos; negligencia benigna; India; América Latina.

\begin{abstract}
Prime Minister Modi injected a new dynamism in conducting India's external relations assuming an influential role in regional and international issues. Since he was elected, he has travelled to almost all continents, but only visiting Latin America twice under multilateral meetings. Latin America offers diverse opportunities to India regarding food and energy security, investments, and South-South cooperation. However, India shows a benign negligence towards the region. In this article Modi's foreign policy is analyzed with particular interest on Latin America to identify opportunities and obstacles in the bilateral relation.
\end{abstract}

Keywords: Foreign policy; opportunities; obstacles; benign negligence; India; Latin America

1 Universidad Empresarial Siglo 21, Argentina. Investigadora. Doctora en Relaciones Internacionales, Correo electrónico: Paola.Baroni@ues21.edu.ar 


\section{Introducción}

Con el fin de la Guerra Fría comienza un proceso de descentralización a nivel mundial, donde se observa el lento declive de Estados Unidos como superpotencia y la presencia más activa, en diversos ámbitos del sistema internacional, de actores emergentes como China e India.

Luego de una profunda crisis económica-financiera, India decide -a principios de 1990- liberalizar su economía, lo que le permite un crecimiento económico sin precedentes y propicia su despegue internacional. Se inserta en el sistema mundial con el estatus de potencia media, producto de su crecimiento económico, pero también debido a su capacidad militar, el desarrollo de la tecnología y del sector servicios, así como a su fuerza laboral (Efstathopoulos, 2011).

Un Estado es considerado potencia media tanto por sus capacidades materiales como por las inmateriales (Rocha Valencia y Morales Ruvalcaba, 2010), así también como por su capacidad para trascender la geografía y proyectar una cierta influencia regional o global (Efstathopoulos, 2011). La crítica principal hacia India ha sido su actuación vacilante en el escenario internacional, a pesar de la gran cantidad de factores - como su seguridad energética y alimentariaque demandan un liderazgo más fuerte para acelerar su crecimiento económico y mejorar su seguridad interna (Mohan, 2006).

Durante su campaña electoral, Modi expresó la necesidad de trabajar para que la India renaciente tuviera su lugar en el concierto de naciones y en las instituciones internacionales. Se planteó reorientar los objetivos, el contenido y el proceso de la política exterior india y ha buscado alejarse de la tradicional actitud 'reactiva', asumiendo un rol más activo (Bhatia, 2016). Sin embargo, y a pesar de haber prometido rejuvenecer las relaciones con América Latina, no se ha desarrollado una política específica para la región.

Hasta el momento, las vinculaciones entre ambas están impulsadas por la diplomacia económica y aunque el intercambio comercial y las inversiones han crecido, aún se encuentran por debajo de su potencial. América Latina ofrece diversas oportunidades a India en términos de seguridad alimentaria (aceite vegetal, granos, azúcar de caña) y energética (petróleo), de desarrollo industrial (litio y cobre), y de la cooperación Sur-Sur. Existe un margen importante para el crecimiento de la cooperación energética, del sector automotriz y de servicios como así también en los recursos naturales. Pero para que los vínculos progresen, es necesario un mayor impulso político por parte de ambos actores (Kasturi, 2016). 
El objetivo del trabajo es caracterizar la política exterior del gobierno de Modi y, a la luz de esta, analizar la relación con América Latina, en busca de establecer oportunidades y obstáculos en las vinculaciones actuales, sobre todo en la dimensión comercial y de cooperación.

El análisis se desarrolló mediante una investigación descriptiva y una estrategia metodológica cualitativa (Valles, 1999). Se realizó la recopilación e interpretación de datos - principalmente secundarios (estadísticos, documentales y periodísticos)-, explorando la información disponible y las relaciones no observables entre los datos (Vieytes, 2004). Se emplearon la técnica de observación documental y la técnica de análisis de documentos de material escrito. Se seleccionaron aquellos textos que dieron cuenta del fenómeno en estudio en un determinado lugar y tiempo ${ }^{2}$. Dentro de esta estrategia se utilizó la triangulación de datos con el propósito de contrastar tanto los datos cuantitativos como cualitativos (Valles, 1999) y se evaluó, así, la convergencia o divergencia de la información recogida.

\section{Abordaje teórico: India, potencia media emer- gente y política exterior}

El contexto global del siglo XXI se caracterizó por ser menos restrictivo que el de la década de 1990 y, por lo tanto, los países experimentaron un mayor grado de libertad para la profundización o el cambio en sus respectivos modelos de desarrollo e inserción internacional (Soares de Lima y Milani, 2016). El sistema internacional del siglo XXI permitió el surgimiento de las potencias medias emergentes, como China, Rusia, Brasil e India, las cuales tienen el capital, la tecnología y las empresas - públicas y privadas- necesarias para generar una inserción internacional más activa.

Según González Levaggi (2016), los Estados emergentes

... se destacan por poseer una creciente estatura económica, un rol destacado a nivel regional como actores claves para la estabilidad y el desarrollo de su área circundante, la búsqueda de un nuevo status que balancee entre las capacidades desarrolladas y el prestigio buscado, la utilización de herramientas de poder suave para atraer a diferentes actores internacionales y, por último, un progresivo empoderamiento en la escena internacional. (p. 28)

2 La técnica de análisis de documentos busca los principales datos internos (datos de autoría, sus orientaciones, fechas y lugares, etc.) y externos (relaciones entre el documento y su medio cultural) que surgen del documento. 
Para Pinar Tank (2012) (como se citó en González Levaggi, 2016), estos países cambiaron la dinámica de la política internacional a través de la búsqueda de una mayor participación en organismos y foros internacionales, y de una interrelación política más profunda en dichas organizaciones como así también en grupos como IBSA, BRICS o MIKTA.

Desde fines de la Guerra Fría, India evidenció la preeminencia de su estatus de poder a través de su crecimiento económico, su capacidad militar, la producción de tecnología y exportación de esta, la proyección hacia la región, entre otros. A las capacidades materiales de India, se suma su poder blando -capacidades inmateriales (Rocha Valencia y Morales Ruvalcaba, 2010) - construido con base en los ideales de los padres fundadores del país, es decir, ser la mayor democracia del mundo que fundamenta sus relaciones exteriores en los principios del no alineamiento, la no interferencia en los asuntos internos de otros Estados y la cooperación internacional para lograr el beneficio mutuo (Baroni y Dussort, 2017).

Este tipo de poder ha sido esencial para India, ya que el gobierno de Modi ha trabajado para acentuar la dimensión cultural en la redefinición de la política exterior de este país. En este sentido, el yoga, la medicina ayurveda, la diáspora india y la religión adquirieron un lugar predominante en la agenda externa; los dos primeros son los de mayor relevancia en la relación con la región latinoamericana (Baroni, 2019).

Los intereses nacionales nutren las decisiones y acciones de política exterior de los Estados. En el caso de India, el marco de las decisiones de política exterior está condicionado por los principios reguladores de la democracia liberal y el pluralismo religioso, étnico y cultural; así como por los objetivos de erradicar la pobreza y asegurar el crecimiento económico y el desarrollo del país. Por eso, ha buscado acercarse a países y regiones que favorecieran el alcance de estos objetivos (Tharoor, 2012). Sin embargo, y a diferencia de China que se proyectó a casi todos los continentes, desde su independencia, India centró su prioridad en la región asiática (Baroni, 2019).

Es importante recordar que la política exterior resulta de un esfuerzo de compatibilizar, a través de una evaluación pragmática de los recursos de poder que se dispone, las necesidades internas con posibilidades externas. Es una herramienta con la que cuentan los Estados y que se diseña desde la guía de los intereses nacionales (Lafer, 2002).

Dentro de la política exterior, los Estados cuentan, entre otras estrategias, con la diplomacia económica para alcanzar sus objetivos, la cual puede definirse como la sumatoria de acciones, procesos, prácticas e instrumentos de naturaleza 
económica -facilitación de comercio, cooperación técnica, estrategias de internacionalización de empresas- emanadas hacia el exterior del Estado, mediante los cuales se crean y distribuyen los beneficios de las relaciones económicas internacionales (Bayne y Woolcok, 2017). En el caso de América Latina, la diplomacia económica ha sido el principal instrumento en el acercamiento hacia la región.

Por otro lado, la política exterior de India buscó -en la persecución de sus objetivos nacionales- el fortalecimiento de las vinculaciones con países en desarrollo, bajo la mirada de la cooperación Sur-Sur. Este tipo de cooperación es la promovida por los países del Sur o por los organismos multilaterales o regionales de los que forman parte. Implica la transferencia de recursos financieros o intercambio técnico desde un país del Sur hacia otro, para así promover el desarrollo económico y social (Lengyel y Malacalza, 2011).

\section{Características de la política exterior de India}

En el actual contexto internacional, India se presenta como un actor central ya que es una de las economías más dinámicas del globo, con un crecimiento del 6,1 \% (2018-2019) (Departamento de Comercio, 2019), superior a la media mundial $^{3}$. Además, ejerce un activo rol en los organismos multilaterales y busca reformarlos - para generar un sistema más justo y equitativo-, y enriquecer el orden mundial (Ragi, Sondhi y Pathak, 2018).

La política exterior de India a partir de la independencia (1947) tuvo, como ejes, el desarrollo de una economía autosuficiente y disminuir el elevado nivel de pobreza, a través de un gobierno democrático. Para este fin, se implementó una estrategia socialista basada en la intervención estatal, en el desarrollo de la industria pesada, en una política de sustitución de importaciones y el control financiero (Baroni y Dussort, 2017). Sin embargo, el resultado -hacia la década de 1980- fue el aislamiento económico internacional del país (Rajan y Sen, 2000) y un bajo desarrollo económico-comercial (Banco Interamericano de Desarrollo [BID], 2010).

Estas condiciones se conjugaron con la caída de su principal aliado, la Unión Soviética, y con las repercusiones en la economía de este país por la Guerra del Golfo (1991), como fue el aumento del precio internacional del petróleo ${ }^{4}$. Esta situación llevó a la entrada en default en 1991, lo que condujo a un programa

3 Según datos del FMI, el crecimiento promedio de la economía mundial para el 2018 fue del 3,6\% y del 3\% para el 2019.

4 Para información sobre la dependencia energética de India, ver U.S. Energy Information Administration (https://www.eia.gov/international/overview/country/IND). 
de ajuste estructural del FMI (Rajan y Sen, 2000) y a redefinir su lugar en el mundo para salir de su aislamiento y adaptarse al nuevo sistema internacional.

Estos eventos llevaron al gobierno de Narsimha Rao (1992-1997) a profundizar e intensificar el proceso de liberalización y apertura económica, favoreciendo, entre otros, el desarrollo de los sectores de informática y telecomunicaciones. Estos procesos de liberalización y apertura económica llevaron a un fuerte crecimiento económico, la caída de la pobreza, así como la mejora de varios índices de desarrollo humano y de diversas actividades económicas (Pérez Llana, 2013).

Dichas reformas implicaron la reformulación de la política exterior y comercial, guiadas ahora por el interés nacional de crecimiento económico y desarrollo del país. Para el gobierno indio era central encontrar socios comerciales que generaran nuevas oportunidades para los productos e inversiones indias (Bhattacharjee, 2012).

Históricamente, India ha desarrollado su política exterior a través de la división del mundo en círculos concéntricos (Seshasayee, 2020). El primer círculo lo forma su vecindario inmediato, Asia del Sur, donde busca primacía y evitar la injerencia de otras potencias. El vecindario extendido (Sudeste de Asia, el Este de Asia, Asia Central, Medio Oriente y el Océano Índico) es el segundo círculo, y aquí busca incrementar su presencia y balancear la influencia de otras potencias, como China. El último círculo incluye el resto del mundo, donde trata de cumplir un rol de potencia emergente a través de su accionar respecto a la paz y a la seguridad internacionales. Se destacan en este último, socios estratégicos como los Estados Unidos y Rusia (Mohan, 2006).

Teniendo en cuenta esta visión y que los países priorizan las regiones y Estados que afectan directamente sus intereses nacionales, se comprende que las vinculaciones con Latinoamérica no hayan sido más profundas.

El nuevo escenario internacional de comienzos del siglo XXI implicó para India reformular sus estrategias diplomáticas hacia una más realista y pragmática (Mohan, 2006). Sin embargo, el gobierno de M. Singh (2004-2014) enfrentó problemas internos por la lentitud de las reformas domésticas, lo que hizo a India vulnerable a los impactos de la crisis mundial de 2008. Esto afectó la eficiencia y el desarrollo de la política exterior hasta la llegada de Narendra Modi al poder en 2014, quien le inyectó nueva energía (Mohan, 2015). 
S. Jaishankar (como se citó en Parameswaran, 2015) plantea cinco innovaciones en la política exterior a partir de Modi.

En primer lugar, una nueva narrativa como parte de la transición de India hacia una potencia líder. Esto se observa en el récord de participación en operaciones de mantenimiento de la paz o en su posición respecto a un asiento permanente en el Consejo de Seguridad.

En segundo lugar, la creación de un nuevo léxico e imaginario, donde se incluye, por ejemplo, las iniciativas Neighbourhood First, Act East y Look West5. Además, la preferencia por el uso del hindi para así proyectar la imagen de una nación orgullosa de su herencia y cambiar la visión de un país modesto y servil a las potencias (Sharma y Miklian, 2016).

Tercero, S. Jaishankar (como se citó en Parameswaran, 2015) plantea el énfasis puesto en el uso del poder blando, el cual puede observarse en el Día Internacional del Yoga y en sus vinculaciones con la herencia y la cultura indias. Cuarto, destaca el rol dado a la diáspora en la construcción de esta nueva imagen, la cual genera un involucramiento más fuerte con los pueblos indios de ultramar. Y quinto, se ha manifestado, de forma más clara, el fuerte vínculo que el país ha establecido entre diplomacia y desarrollo nacional.

A través de estas innovaciones, el objetivo primordial de la gestión de Modi ha sido transformar la política exterior y lograr que sea una herramienta que ayude a hacer de India una potencia global. A este respecto, ha sido necesario fortalecer las vinculaciones regionales y que la política económica lidere los lineamientos de su política exterior (Rao, 2016).

En los últimos años, India ha buscado fortalecer sus vinculaciones con Estados Unidos, Medio Oriente, China, el Sudeste de Asia, entre otros; con diferentes niveles de resultados. En el caso de América Latina, ha estado casi ausente, aunque la región se está convirtiendo en un actor importante, fundamentalmente en la dimensión económico-comercial (Desai, 2015).

5 La primera refiere a los países vecinos y a las principales potencias asiáticas; la segunda es la continuidad de la Look East Policy, vinculada al este y sudeste asiático. La tercera busca profundizar la relación con Medio Oriente y el Golfo Pérsico. 


\section{Vinculaciones históricas entre India y América Latina}

La distancia geográfica, la falta de vínculos históricos, la brecha lingüística y las diferentes tendencias políticas (Ross, 2010), junto a las prioridades dadas a ciertos temas internos y regionales y a que América Latina no afectaba su seguridad, son algunas de las causas de la escasez de vinculaciones con la región. Por su parte, Asia tampoco ha sido -hasta el siglo XXI- el centro de atención de la política exterior de los países latinoamericanos.

Los primeros contactos fueron con la zona del Caribe durante el siglo XIX, cuando los indios iban a trabajar en las plantaciones británicas. Aunque se produjo la apertura de embajadas a partir de la independencia, las primeras visitas de Estado se realizaron recién en la década de $1960^{6}$. Además, las misiones diplomáticas indias resultaban insuficientes -en cantidad y en capacidades específicas- para estimular el comercio y las inversiones (Ross, 2010).

La mayor vinculación política se dio en asuntos multilaterales afines al Tercer Mundo, el desarrollo y el nuevo orden internacional (visión sur-sur) y, a nivel bilateral, la principal expresión del vínculo fue la comercial. Al ser economías similares -producían recursos naturales, materias primas y manufacturas asociadas a estos sectores-, el intercambio comercial fue limitado hasta la década de 1990. Por otro lado, existía una brecha tecnológica - a favor de Brasil, Argentina y México-, lo que imposibilitó el comercio de otros bienes, como los de capital (Ross, 2010).

Las políticas de India hacia países individuales de la región no mostraron una variación significativa a lo largo de las décadas, con excepción de Brasil que tuvo un rol destacado tanto antes como después de la liberación de Goa del dominio portugués (1961) (Sahni, 2015). Según Gangopadhyay (2014) y Bhojwani (2015), esta situación responde, en gran parte, a la percepción india de América Latina como una región más o menos homogénea a nivel político, económico y social, sin tener en cuenta sus diversas complejidades.

La llegada de India a la región latinoamericana es, entonces, tardía, a posteriori de la apertura e inserción del país surasiático en el escenario comercial internacional. Sahni (2011) identifica diversos intereses al acercarse a Latinoamérica en este nuevo contexto; entre ellos destaca la necesidad de contar con una

6 México en 1961 -realizada por Nehru-; y Brasil, Argentina, Chile, Uruguay, Colombia, Venezuela y Guyana en 1968, liderada por Indira Gandhi. 
presencia mayor en diferentes partes del globo -vinculada al fortalecimiento de su posición de potencia media emergente-; la comunión de intereses de los países de la región en los foros multilaterales y finalmente, acercar posiciones a su principal competidor en la escena asiática - China- que cuenta con acciones desde hace más de dos décadas en la región latinoamericana (Baroni, 2015).

En la dimensión comercial se ha concentrado la mayor actividad de vinculación. Con el objetivo de insertarse en la economía internacional -en un contexto de creciente interdependencia comercial y financiera- India plantea una política exterior hacia la región "con énfasis en la relación rentable y no en la retórica y el simbolismo" (Sahni, 2011, p. 172).

En este sentido, una de las herramientas utilizadas por India ha sido la diplomacia económica, con la que busca promover el comercio exterior y las inversiones (BID, 2010). Así, el acercamiento - desde fines del siglo XX-, ha tenido como eje central las vinculaciones comerciales, pero ahora con base en un patrón interindustrial: exportación de productos primarios y manufacturas de recursos naturales por parte de América Latina y la importación de bienes de tecnología alta y media desde India.

Entonces, el cambio a una política de compromiso estratégico con la región coincide con la emergencia de India en el sistema internacional (Sahni, 2015) y se entiende en el marco de los cambios sistémicos -como la transición del poder de Occidente a Oriente- y en los intentos de India de enfrentar las consecuencias que genera ser una economía emergente, entre ellas, la seguridad energética y la seguridad alimentaria (Sahni, 2015). Sin embargo, se critica la falta de una visión específica por países, basada en la identificación de temas y áreas de cooperación (Gangopadhyay, 2014).

\section{La política exterior hacia América Latina}

La estrategia de vinculación con la región, diseñada a finales de la década de 1990, se sostiene hasta el momento sin mayor innovación. El eje principal sigue siendo el comercial, lo que llevó a un crecimiento del intercambio y a las inversiones, aunque siguen estando por debajo de su potencial (Estevadeordal et al., 2017). 


\section{Dimensión política}

El establecimiento de misiones diplomáticas permanentes es importante para desarrollar las relaciones entre Estados. Existen 14 representaciones indias en la región latinoamericana (2020), lo cual es insuficiente, si se tiene en cuenta que América Latina y el Caribe comprende más de 40 países. Esto limita el conocimiento mutuo y la posibilidad de fomentar los vínculos, por lo que es necesaria la apertura de embajadas en países considerados importantes, por ejemplo, Ecuador o República Dominicana, quienes están entre los principales socios comerciales (ver Tabla 1).

Desde su asunción, Modi ha viajado a casi todos los continentes en varias ocasiones. Sin embargo, ha realizado solo dos visitas de Estado en marcos multilaterales a América Latina ${ }^{7}$, es decir, no ha realizado una visita oficial a nivel bilateral al continente. Las visitas oficiales del principal líder del Ejecutivo -en el caso de India el Primer Ministro- implican dar importancia a las relaciones con el país receptor. En el caso de América Latina, la subsistencia de una visión simplista y homogénea de la región sigue dificultando un correcto acercamiento (Bhojwani, 2015).

No obstante, se observa un incremento de las visitas bilaterales de alto nivel por parte de presidentes y vicepresidentes de India hacia América Latina, aunque la repercusión de estas es menor. El gobierno indio, además, ha desarrollado mecanismos institucionales para revisar los vínculos políticos, comerciales y de inversión y ha creado áreas comerciales y de marketing en las misiones diplomáticas (Departamento de Comercio, 2019).

Es importante destacar que también se observa un importante incremento en las visitas de alto nivel (presidentes) desde la región latinoamericana hacia India, por ejemplo, desde Chile, Colombia, Paraguay, Venezuela, Argentina, y varias desde Brasil. Esto indica la intención de estos países de profundizar los vínculos con India (Seshasayee, 2020). Durante estas visitas se revisa la relación bilateral y la cooperación en áreas trascendentes como energía, agricultura, informática, industria automotriz y farmacéutica, entre otras.

Dentro de la dimensión política, se destaca el impulso dado a la cooperación sur-sur con el fin de contribuir al progreso tanto de la región como de India. A nivel de ayuda oficial al desarrollo, América Latina recibe menos del $1 \%$ de la otorgada por India; sin embargo, este tipo de cooperación se ha desarrollado a través de acuerdos sobre diferentes temáticas como las tecnologías de la

7 La cumbre de los BRICS en Brasil en 2014 y la Cumbre del G20 en Argentina en 2018. En 2016 realizó una breve visita de trabajo a México de solo 4 horas, en viaje a EE.UU. 
información, la educación, la reducción de la pobreza, la cooperación antártica y energética; entre otras (Comisión Económica para América Latina y el Caribe [CEPAL], 2016). No obstante, se avanzó poco en la implementación de estos acuerdos. La mayoría de los compromisos tardaron en concretarse y muchos de ellos quedaron en letra muerta.

Se ha observado también la participación de India en ámbitos regionales específicos, como en el Sistema de Integración Centroamericana (SICA) -a través de un mecanismo institucional para el diálogo-, o en la Comunidad de Estados de Latinoamérica y el Caribe (Celac), para trabajar sobre una agenda que incluye temas como energía, agricultura, medicina, astronomía, entre otros (Siddiqui, 2015). Además de lo expuesto, se destaca la inclusión, por parte de India, de la mayoría de los países de la región en el programa de visa electrónica para turistas (e-Tourist Visa), lo que hace más ágil el proceso.

Las potencias medias también utilizan el poder blando en su política exterior. En el caso de India se destaca el establecimiento del Día Internacional del Yoga (21 de junio), el cual es celebrado a gran escala en los países de la región con el apoyo de autoridades locales. Esta acción contribuye a reforzar la imagen benévola y positiva que se tiene de India, vinculada a Gandhi, a la medicina ayurveda, a su comida y a su música (Seshasayee, 2020).

Sin embargo, se plantea que no ha utilizado de forma eficiente este tipo de poder, ya que no ha logrado - excepto en Brasil- el desarrollo de interacciones académicas (casi inexistentes), la comercialización de su industria cinematográfica y la eficiencia de programas y becas culturales y técnicas (Bhojwani, 2017).

Dentro de este contexto, la excepción ha sido Brasil. Las semejanzas entre los dos países -extenso territorio, una gran población multirracial y multirreligiosa y variedad de recursos-, fue central en la identificación de intereses en común, lo que generó una mayor intensidad en las vinculaciones. Estas deben comprenderse desde una mirada bilateral y sistémica ya que, al ser potencias emergentes, han desarrollado una conciencia similar en diversos temas de la agenda internacional. La convergencia en foros multilaterales como BRICS, IBSA ${ }^{8}$ y G-20 es un claro ejemplo, ya que buscó sumar poder, solucionar problemas comunes y construir comunidad, a través de la coordinación de posiciones y acciones (Sahni, 2015).

8 BRICS: término acuñado por economistas en Goldman Sachs en 2003, hace referencia a las economías combinadas de Brasil, Rusia, India, China y Sudáfrica (incorporada en 2011).

IBSA: foro trilateral creado por India, Brasil y Sudáfrica en 2003, con el objetivo de promover la cooperación comercial, las inversiones y la diplomacia económica entre los miembros. Este surge ante el fracaso de las rondas de negociaciones de la OMC en Cancún. 
A partir del nuevo milenio se profundizaron las relaciones a través de la multiplicación de los contactos de negocios y políticos de alto nivel, que estimularon las oportunidades de relacionamiento y cooperación. Esto generó implicancias estratégicas para ambos (Sahni, 2015), que se materializaron en el establecimiento de una Asociación Estratégica en 2006.

Por su parte, México se ha sumado a esta tendencia, pues es también una economía emergente con similares miradas respecto al desarrollo. Se ha convertido en uno de los principales socios comerciales de India en la región; su proximidad al mercado estadounidense y la integración industrial con dicho país ha sido central en este acercamiento (Seshasayee, 2020).

\section{Dimensión comercial}

En el periodo 2018-2019, la región latinoamericana tuvo una participación del $3,59 \%$ en el total del comercio externo indio. Este porcentaje muestra una disminución respecto a períodos anteriores ${ }^{9}$ (Departamento de Comercio, 2019) porque se experimentó una fuerte recesión en varias de las economías de la región latinoamericana y se produjo la caída de los precios internacionales de los productos básicos exportados.

Las exportaciones de India - con una tendencia creciente- también disminuyeron, pasando de un 4,02 \% en 2013-2014 a un 2,69 \% en el 2018-2019 (Departamento de Comercio, 2019). Esta desaceleración se explica por la caída del comercio internacional y de la demanda de Brasil, por las crisis de Venezuela y Argentina, como también por la diversificación de proveedores que ha logrado India en el tipo de productos que importa desde América Latina.

Como las importaciones han crecido más que las exportaciones hacia Latinoamérica, se observa una balanza comercial sostenidamente deficitaria para India. Lo que sobresale es que pocos Estados de la región han focalizado la atención (ver Tabla 1), por lo que se evidencia una concentración de socios, pero a la vez la posibilidad de diversificar los vínculos. Además, se evidencia que México se convirtió en el principal socio comercial en 2018-2019 y se constituye, junto a Brasil, en un importante socio político y comercial.

9 El proceso de disminución de la participación de América Latina en el comercio exterior de India se observa a partir del período 2014-2015 (con un 5,07 \%): 3,92\% en 2015-2016; 3,71 \% en 2016-2017; 3,62 \% en 2017-2018 (Trade Statistics, Department of Commerce https://tradestat.commerce.gov.in/ eidb/default.asp). 
Tabla 1

Principales socios comerciales de India en América Latina y el Caribe (2018-2019)

\begin{tabular}{llccc}
\hline \multicolumn{1}{c}{ País } & Exportaciones & Importaciones & Comercio total \\
\hline 1 & México & 3.841 & 5.577 & 9.418 \\
2 & Brasil & 3.800 & 4.406 & 8.206 \\
3 & Venezuela & 165 & 7.259 & 7.424 \\
4 & Perú & 721 & 2.405 & 3.126 \\
5 & Argentina & 563 & 1.955 & 2.518 \\
6 & Chile & 990 & 1.238 & 2.228 \\
7 & Colombia & 1.117 & 1.055 & 2.172 \\
8 & Bolivia & 105 & 852 & 957 \\
9 & R. Dominicana & 216 & 567 & 783 \\
10 & Ecuador & 298 & 219 & 517 \\
\hline
\end{tabular}

Nota: Cifras expresadas en millones de dólares. Elaborado en base a datos del Departamento de Comercio de India, 2019.

Es importante considerar en el análisis el menor papel de India en el comercio internacional, ya que cuenta con una apertura comercial reducida y elevados niveles de protección, sobre todo, en el sector agrícola (BID, 2010). El arancel promedio de este sector fue del 36,4 \% (en 2014-2015) mientras que el de China fue del $14,8 \%$ y las tasas más altas se aplicaron a los productos en los que la región tiene ventaja comparativa (Estevadeordal et al., 2017).

También se aplicó otro tipo de medidas como: a) contingentes arancelarios a productos agrícolas a partir de una cierta cantidad; b) ajuste de aranceles, con alícuotas más elevadas; c) aranceles ad valorem al $6 \%$ de las líneas arancelarias; y d) medidas sanitarias y fitosanitarias e impedimentos técnicos. Como resultado, se observa una elevación de los costos y una mayor incertidumbre para el ente exportador (Estevadeordal et al., 2017).

Esta situación dificulta el comercio con América del Sur, debido al patrón de intercambio comercial interindustrial. Sin embargo, esto no sucede con México y los países centroamericanos, que compiten respecto a algunos productos manufacturados e industriales como textiles, autopartes o electrónica (CEPAL, 2016). En este sentido, las alícuotas tienden a disminuir en los bienes con mayor grado de procesamiento (Estevadeordal et al., 2017). 
A pesar de los elevados aranceles, commodities es la partida que más ha crecido y la que mayor impacto tiene en la balanza comercial. Los principales productos son el petróleo (Venezuela, México, Brasil, Colombia, Ecuador); el oro (Perú, Bolivia, Brasil, República Dominicana, Colombia); el aceite vegetal comestible (Argentina y Brasil), y el cobre (Chile) (UN Comtrade, 2019). América Latina provee casi el $20 \%$ del petróleo que importa India y el $22 \%$ del aceite vegetal (Departamento de Comercio, 2019). Se observa, entonces, una alta concentración en la canasta exportadora latinoamericana, compuesta por escasos productos con nulo o bajo valor agregado.

Respecto a las exportaciones de India hacia la región, los principales productos son los vehículos (3 231 millones de dólares); los productos químicos -insumos para la industria textil, alimentaria y química - (2 576 millones de dólares); maquinaria (1 426 millones de dólares); textiles (1 058 millones de dólares); diésel (1 049 millones de dólares); productos farmacéuticos (962 millones de dólares); hilados de algodón (359 millones de dólares); plásticos (386 millones de dólares), entre otros. América Latina explica el $28 \%$ de las exportaciones globales indias de automóviles, donde México es el principal destino y el $22 \%$ de las exportaciones de motocicletas (Departamento de Comercio, 2019).

Este tipo de intercambio - similar al que se tiene con otros países asiáticosgenera una nueva forma de subalternización de los países latinoamericanos, dependiendo, ahora, de los precios internacionales de los commodities -sujetos a condiciones externas de demanda y producción (factores climáticos, sociales y políticos)- (Nacht, 2013), y de los capitales extranjeros en áreas como la minería y los agronegocios (Frechero, 2013).

La estrategia de acercamiento utilizada ha sido la diplomacia económica que se materializó en el programa FOCUS: Lac, creado en 1997 con el objetivo de intensificar las exportaciones indias hacia la región. El programa ofrece asistencia financiera a empresas indias para participar en exposiciones comerciales y misiones comerciales, y para realizar estudios de mercado. También financia el viaje a personal empresario latinoamericano para que asista a eventos y exposiciones comerciales en la India (Viswanathan, 2008).

Este programa se complementa con la realización de cónclaves de negocios e inversiones, organizados por la Confederación de Industria de India, en colaboración con el gobierno indio. Los encuentros son plataformas de contacto entre los diversos actores de ambas regiones, vinculados a las temáticas económicas, comerciales y financieras. 
Otro instrumento de las relaciones comerciales son los acuerdos comerciales; sin embargo, India se ha mostrado reticente a utilizarlos, optando por la red Acuerdos de Alcance Parcial (BID, 2010). Así, con la región cuenta con un Acuerdo de Preferencias Fijas con el Mercosur, firmado en 2004 y vigente desde 2009 y un Acuerdo Preferencial Parcial con Chile (2007). Respecto al primero, India otorgó preferencias comerciales a 450 líneas arancelarias y el Mercosur a 452. Los principales sectores incluidos son: carne y productos cárnicos, productos químicos inorgánicos y orgánicos, colorantes y pigmentos, cueros y pieles, lana, hilo de algodón, maquinaria y equipos eléctricos, preparados alimenticios, productos farmacéuticos, plásticos y sus manufacturas, caucho y productos de caucho, entre otros.

En 2010 comenzaron las negociaciones para expandir el acuerdo; pero, recién en 2016, se intercambiaron las listas de productos para ampliarlas a 3000 . La dificultad en la negociación radica en la resistencia de los sectores industriales de Brasil y Argentina (La Vanguardia, 2016) y en el sector agrícola indio. Este acuerdo tiene un impacto ínfimo, pues es limitado en la cobertura $-3,2 \%$ de las líneas-y profundidad de la liberalización; es decir, casi no da ninguna preferencia en el mercado indio (Estevadeordal et al, 2017).

Respecto al segundo caso (Acuerdo Preferencial con Chile), se negoció la ampliación y profundización del acuerdo de 2007 -también muy limitado-, pasando de 474 productos a casi 2 800. Los principales sectores son el silvoagropecuario, la industria maderera, la carne y productos cárnicos, el cobre, productos químicos orgánicos e inorgánicos, productos farmacéuticos, colorantes y pigmentos, entre otros. El nuevo acuerdo (2014) fue ratificado recién en 2016 debido al fuerte lobby de agricultores indios y, según Bhojwani (2015), a una muy lenta burocracia. En 2019 se acordó avanzar en una nueva ampliación, y se realizó, en diciembre de ese año, la primera reunión (Embajada de Chile en India, 2019).

India también está analizando un acuerdo de libre comercio con Perú -que lleva 5 rondas de negociaciones-, el cual incluiría el comercio, la inversión, servicios y relaciones culturales (Ministerios de Asuntos Externos de India, 2020). Además, se ha acercado a la Alianza del Pacífico y ha sido admitida -en 2014- como Estado Observador (Departamento de Comercio, 2019).

Un actor central de la inserción comercial de India ha sido su sector externo. Aunque la inversión extranjera directa (IED) en América Latina no es significativa, se estima en unos 14 mil millones de dólares (Departamento de Comercio, 2019). Los principales sectores son el farmacéutico, el autopartista, la minería, los hidrocarburos, los recursos naturales, las tecnologías de información (TI) 
y sus respectivos servicios ${ }^{10}$. Lo importante a destacar es que la mayoría de las inversiones indias contribuyen al desarrollo de una mano de obra calificada y a la transferencia de tecnología (Ellis, 2015).

Por su parte, las políticas indias sobre la IED han tendido a la restricción en sectores como la agricultura y los servicios, donde se insertan las multilatinas y responden a regulaciones muy burocráticas. No obstante, el gobierno indio ha implementado medidas que flexibilizan algunas restricciones y simplifican procedimientos administrativos (Estevadeordal et al., 2017).

Por otro lado, y a diferencia de otros actores internacionales presentes en la región (Europa, China, Japón, etc.). India no participa en las instituciones financieras regionales como el BID o la Corporación Andina de Fomento (CAF). Esta situación no le permite ser partícipe, por ejemplo, en los diversos proyectos de infraestructura que se llevan a cabo en la región (Bhojwani, 2017).

Entonces, la brecha entre el comercio potencial y el real entre India y América Latina obedece a cuestiones que no tienen ver con las ventajas comparativas. Según el BID (2010), el comercio está muy distorsionado y la limitación tiene su base en los altos costos de las transacciones comerciales, en las barreras arancelarias y no arancelarias y en los costos de transporte. A esto se suma la falta de infraestructura en ambas partes ${ }^{11}$, la falta de conectividad -escasez de puertos de gran calado y aeropuertos-y la ausencia de líneas de transporte marítimo y aéreo directo.

En los últimos años ha sido evidente que la identificación de los países de la región con mayor impulso llevó a inclinarse hacia los Estados más grandes -Brasil y México-, ya que proveyeron de diferentes oportunidades políticas y económicas. A pesar de que se identificaron temas de común preocupación y se proyectaron acciones, el comercio siguió siendo la principal dimensión de la política de India hacia la región (Gangopadhyay, 2014).

10 Se destacan: Tata Consulting Services, Cognizant Technologies y Cellent (TI y servicios); Bajaj (motocicletas); United Phosphorus Ltd (agroquímicos y semillas); Glenmark, Dr.Reddy's, Seven Pharma (productos farmacéuticos); Godrej (productos cosméticos); Arcelor Mittal (acero); Jindal Steel Works (minería); Tata Motors, Mahindra \& Mahindra, Hero Motocorp (automóviles y autopartes); ONGC Videsh Limited (hidrocarburos), entre otras.

11 Por ejemplo, la falta de conectividad al interior de India (falta de carreteras y vías férreas) y la poca conectividad entre la costa atlántica y la pacífica de Latinoamérica. 


\section{Oportunidades}

El tipo de intercambio interindustrial entre ambas regiones afecta el desarrollo de los países latinoamericanos, ya que los hace dependientes de los precios internacionales de los commodities y de los productos agrícolas, lo cual afecta su desarrollo industrial. Como la demanda india seguirá sosteniéndose en estos sectores, ya que necesita satisfacer las demandas de una población -y clase media- en aumento y mantener su crecimiento económico, se hace necesario incluir otros bienes con mayor valor, como los derivados del cobre o del petróleo o manufacturas de origen agropecuario, aprovechando las ventajas comparativas existentes (Estevadeordal et al., 2017).

Se observa, por otro lado, una complementariedad potencial en el área de servicios como también en el sector automotriz. En el primer caso, América Latina no solo ofrece recursos humanos calificados -Argentina, Chile, Brasil, México-, sino también una plataforma estratégica para la exportación de productos gracias a la proximidad geográfica con Estados Unidos y a los diferentes husos horarios de la región (Estevadeordal et al., 2017). Aunque es el sector en donde más inversiones indias hay, es necesaria la expansión hacia una mayor diversidad de productos exportables.

En el segundo caso, India puede insertarse en las cadenas de valor integradas, como la que constituyen Brasil, Argentina y México. Aunque se han manifestado intenciones respecto a inversiones en el sector (a través de adquisiciones o joint ventures), hasta el momento no ha habido avance en el tema (Seshasayee, 2020), y el comercio se ha desarrollado a través de exportaciones intrafirma ${ }^{12}$. En el caso de las motocicletas, las empresas indias sí han invertido en la región, ya sea a través de joint ventures (Argentina) o de inversiones directas (Colombia).

De lo expuesto se desprenden dos cuestiones: primero, que deben incrementarse los acuerdos comerciales con los países de la región y profundizarse los existentes, ya que generarán oportunidades y condiciones favorables para el comercio y las inversiones; y segundo, que se debe avanzar en la colaboración efectiva con los grupos regionales, como la Alianza del Pacífico o el SICA (Estevadeordal et al., 2017; Seshasayee, 2020).

Respecto al área de cooperación, se ha observado la firma de diversos instrumentos, aunque varios de ellos no se han puesto en marcha aún. La cooperación en temas energéticos, de salud o agrotecnológicos tiene gran potencial. En

12 Es el comercio que se realiza entre las empresas subsidiarias de una misma empresa transnacional, como son Toyota, Ford y General Motors que tienen plantas en la India. 
el primer caso, la cooperación en energías renovables -muy desarrolladas en América Latina- es importante para India, debido a su dependencia energética. India puede participar en proyectos de energía eólica o solar en la región -para adquirir know how-, como así también en la explotación y fabricación de baterías de litio - para vehículos eléctricos-, ya que es un nuevo recurso energético estratégico $^{13}$ (Seshasayee, 2020).

Respecto al segundo caso, se hace referencia a la cooperación en biotecnología o medicina nuclear (Seshasayee, 2020). Varios países de la región, como Brasil y Argentina, tienen un importante desarrollo en estos ámbitos -incluida el área farmacológica- como así también en el sector de aparatología médica ${ }^{14}$. Entonces, se pueden promover investigaciones e inversiones conjuntas, con claros beneficios para ambas regiones.

En el tercer caso, se plantea la adquisición de tecnología, por parte de India y la transferencia de tecnología sobre diversos aspectos de la producción agrícola, como el riego, las semillas, la maquinaria agrícola, la agricultura de precisión, entre otros (Estevadeordal et al., 2017). La experiencia y el desarrollo alcanzado por los países de la región, en estos temas, generan un gran potencial de cooperación.

En el sector aeroespacial existe una incipiente cooperación, fundamentalmente en el área satelital: monitoreo de satélites; estudios climáticos, territoriales y medioambientales; aplicación de teledetección, entre otros que pueden ser ámbitos de profundización de la cooperación. Argentina, Colombia, Brasil y Chile han lanzado satélites desde bases indias y los acuerdos firmados brindan un marco favorable para el desarrollo de las vinculaciones (Seshasayee, 2020).

Por último, ambas regiones tienen desafíos comunes en los cuales pueden profundizar la cooperación. Existen acuerdos y colaboración -aunque a veces limitados- en temas como el combate de pobreza, aumentar la inclusión financiera, mejorar la gobernanza, entre otros. El intercambio de experiencias -transferencia de conocimiento- en estas temáticas puede generar importantes avances en las sociedades de ambas regiones.

13 Argentina, Bolivia y Chile integran el "triángulo del litio", el cual tiene alrededor del $67 \%$ de las reservas probadas y cerca de la mitad de la oferta global de este mineral (U.S. Geological Service (2018). Mineral commodity summaries 2018. https://s3-us-west-2.amazonaws.com/prd-wret/assets/ palladium/production/mineral-pubs/mcs/mcs2018.pdf)

14 La pandemia puso de manifiesto la necesidad de este tipo de cooperación. En el caso de India, compró respiradores producidos en Argentina y varios países latinoamericanos han adquirido principios activos farmacéuticos desde India y vacunas. 


\section{Reflexiones finales}

América Latina le ofrece oportunidades a India, no solo en términos de seguridad alimentaria y energética, sino también en temas de cooperación Sur-Sur. Al analizar los vínculos, el primer rasgo que sobresale es que el acercamiento no fue planificado. Los cambios producidos en el sistema internacional -con un mayor multilateralismo-y los intereses nacionales permitieron el acercamiento y el aprovechamiento de la vinculación y de los acuerdos a nivel político.

Según Gangopadhyay (2014) y Bhojwani (2015), India presenta una negligencia benigna hacia América Latina y esto se observa en la carencia de una política específica hacia la región que tenga en cuenta las diferentes características de las subregiones. Por otro lado, las Cancillerías latinoamericanas tampoco cuentan con áreas específicas para India, sino que la incluyen en departamentos más generales que abordan toda Asia, Oceanía e islas del Pacífico, aunque la excepción a esto suele ser China.

La dimensión comercial es la que más se ha desarrollado, y aunque el comercio bilateral se ha multiplicado y las inversiones han crecido, están por debajo de su potencial. El comercio está concentrado en un escaso número de países y en pocos productos con escaso valor agregado, como son las commodities y las manufacturas agrícolas. Así, la región latinoamericana se inserta en la economía india a través de su seguridad alimentaria y energética, cumpliendo un rol primario agroexportador que la hace más vulnerable.

Respecto a las inversiones indias, han crecido en sectores significativos como el de TI, el farmacéutico y el de motocicletas, generando puestos de trabajo calificados y transferencia de know how. En este sentido, India debería promover y facilitar las inversiones y exportaciones de sus compañías a través, por ejemplo, de los bloques regionales o acuerdos comerciales.

La complementariedad productiva entre India y la región provee de oportunidades a desarrollar, especialmente, si se toma en cuenta la creciente demanda india de recursos en función del crecimiento de su población y de su desarrollo económico. Sin embargo, también se presentan desafíos en el ámbito comercial.

En el caso de la región latinoamericana, el desafío radica en diversificar su oferta exportable hacia productos con mayor valor (biotecnología, productos y servicios médicos, bienes tecnológicos, etc.) y lograr esquemas de cooperación tecnológica, científica y energética que permitan continuar y avanzar en la transferencia de tecnología y conocimiento. Además, debe trabajar en la 
generación de cadenas de valor regionales, lo que permitiría una mejor inserción de los países en las cadenas productivas indias.

En el caso de India, debería avanzar en la negociación de acuerdos comerciales, ya sea con países individuales o con el Mercosur, y en una mayor liberalización de su economía. Esto permitirá a sus exportaciones competir en mejores condiciones con productos de terceros Estados y lograr la apertura de mercados a productos indios, con base en la reciprocidad. Por otro lado, la identificación de socios clave es necesaria para adoptar políticas más eficientes respecto a su posición en la región como así también para expandir sus asociaciones tecnológicas y diversificar sus socios comerciales y políticos.

Para ambas partes, el reto está en aumentar los estudios de mercado, el conocimiento recíproco y la difusión de las ventajas mutuas. Además, deben trabajar en el desarrollo de infraestructura y logística y en disminuir los costos comerciales. Esto ayudará a facilitar la profundización de las vinculaciones y, tanto India como América Latina, se percibirán como socios naturales y estratégicos que pueden ayudarse mutuamente en su desarrollo.

Por último, y a nivel de cooperación Sur-Sur, las oportunidades se observan en diversos ámbitos, como el tecnológico, el de salud, el agrícola y el energético, entre otros. Ya se han identificado varias de las áreas de interés mutuo y se han firmado acuerdos de cooperación, por lo que el desafío está en activar la voluntad política para la puesta en marcha de los proyectos. Impulsar y sostener la cooperación Sur-Sur es central para complementar las potencialidades, compartir experiencias y desplegar acciones en conjunto con el fin de promover el desarrollo de las sociedades de ambas regiones.

\section{Referencias}

Banco Interamericano de Desarrollo (BID) (2010). India: Oportunidades y desafíos para América Latina. http://www.iadb.org/intal/intalcdi/PE/2010/05898.pdf

Baroni, P. (22-24 de julio de 2015). El acercamiento de India a la región: Desafíos y oportunidades para América del Sur. Ponencia. En VIII Congreso Latinoamericano de Ciencia Política, Asociación Latinoamericana de Ciencia Política. ALACIP, Lima, Perú. http://files.pucp.edu.pe/sistemaponencias/wp-content/uploads/2014/12/Baroni_Paola_Ponencia_El-acercamiento-de-India-a-la-regi\% $\% 3 \%$ B3n-desafios-y-oportunidades-paraAm\%C3\%A9rica-del-Sur-2009-2014.pdf 
Baroni, P. A. (2019). Política exterior argentina hacia la república de India: Factores políticos y económicos, internos y externos [Tesis doctoral]. Universidad Nacional de Rosario, Rosario, Argentina. https://rephip.unr.edu.ar/ handle/2133/16653

Baroni, P. y Dussort, M. N. (2017). El cambio de la Look East Policy a la Act East Policy en India. Relaciones Internacionales, 26(52), 115-136. https://revistas. unlp.edu.ar/RRII-IRI/article/view/2855

Bhatia, R. (30 de mayo de 2016). A review of Narendra Modi's foreign policy. Newslaundry.com. http://www.newslaundry. com/2016/05/30/a-review-of-narendra-modis-foreign-policy/\#

Bhattacharjee, R. (2012). India and the World. Arihant.

Bhojwani, D. (2015). Latin America and India: Understanding Mutual Opportunities. Indian Foreign Affairs Journal, 10(1), 50-62. http://www.associationdiplomats.org/publications/ifaj/Vol10/10.1/10.1-ARTICLE\%202_P-DB.pdf

Bhojwani, D. (2017). Diplomacia colaborativa. En INTAL Lab-BID (comp.) Latindia. El futuro de la cooperación de India y América Latina (pp. 52-63). BID-INTAL.

Bayne, N. y Woolcok, S. (2017). The New Economic Diplomacy: Decision-Making and Negotiation in International Economic Relations. Routledge.

Comisión Económica para América Latina y el Caribe (CEPAL). (2016). Fortaleciendo la relación entre la India y América Latina y el Caribe. México: CEPAL. https://www.cepal.org/es/publicaciones/40755-fortaleciendo-la-relacion-la-india-america-latina-caribe

Departamento de Comercio. Ministerio de Comercio e Industria de India. (2019). Annual Report 2018-19. https://commerce.gov.in/wp-content/uploads/2020/02/ MOC_637036322182074251_Annual-Report-2018-19-English.pdf

Desai, R. (25 de junio de 2015). A New Era for India-Latin America Relations? Forbes. http://www.forbes.com/sites/ronakdesai/2015/06/25/a-new-era-forindia-latin-america-relations/\#d1432f829d47

Efstathopoulos, Ch. (2011). Reinterpreting India's Rise through the Middle Power Prism. Asian Journal of Political Science, 19(1), 74-95. https://doi.org/ 10.1080/02185377.2011.568246

Embajada de Chile en India. (2019). Relación bilateral. https://chile.gob.cl/india/ relacion-bilateral

Ellis, R. E. (24 de junio de 2015). Es la hora de hablar sobre la India y Latinoamérica. Observanto. Análisis Internacional. http:/www.observanto.com/site/ notas/452/Es-la-hora-de-hablar-sobre-la-India-y-Latinoamerica\%20.html 
Estevadeordal, A., Mesquita, M. y Kahan, T. (2017). Hacia una agenda común de comercio. En INTAL Lab-BID (Comp.) Latindia. El futuro de la cooperación de India y América Latina (pp. 14-32). BID-INTAL.

Frechero, J.I. (2013). Extractivismo en la economía argentina. Categorías, etapas históricas y presente. Estudios críticos del desarrollo, 3(4), 45-82. https://estudiosdeldesarrollo.mx/estudioscriticosdeldesarrollo/wp-content/ uploads/2019/01/ECD4-2.pdf

Gangopadhyay, A. (2014). India-China Competitions in Latin America: Some Observations. Jurnal Global \& Strategis, 8(1), 1-13. http://journal.unair.ac.id/ download-fullpapers-jgs3fleeb7f452full.pdf

González Levaggi, A. (2016). Potencias (re)emergentes hacia un mundo policéntrico: Rusia y Turquía frente a América Latina. Relaciones Internacionales, 25(50), 21-47.

India y Mercosur intercambian listas para ampliar preferencias arancelarias. (29 de julio de 2016). La Vanguardia. http://www.lavanguardia.com/politica/20160729/403555360178/india-y-mercosur-intercambian-listas-para-ampliar-preferencias-arancelarias.html

Kasturi, Ch. S. (16 de mayo de 2016). Friends new or old, Modi short of time -Latin America to Africa, Pacific nations to NAM- all kept waiting. The Telegraph. http://www.telegraphindia.com/1160516/jsp/nation/story_85933.jsp\#. V5ldb4-cHIV

Lafer, C. (2002). La identidad internacional de Brasil. Fondo de Cultura Económica.

Lengyel, M. y Malacalza, B. (16-19 de febrero de 2011). What do we talk when we talk about South-South cooperation? The construction of a concept from empirical basis. En IPSA-ECPR Joint Conference, San Pablo, Brasil.

Ministerio de Asuntos Externos de India. (2020). India-Peru Relations. http://mea. gov.in/Portal/ForeignRelation/Peru_Bilateral_brief_January_2020.pdf

Mohan, R. C. (2006). India and the Balance of Power. Foreign Affairs, 85(4), 1732. Recuperado de https://www.foreignaffairs.com/articles/asia/2006-07-01/ india-and-balance-power

Mohan, R.C. (2015). Foreign Policy after 1990: Transformation through Incremental Adaptation. En D. Malone, C. R. Mohan, Sr. Raghavan (Eds.), The Oxford Handbook of Indian Foreign Policy (pp.175-191). Oxford University Press

Mohan, C. R. (5 de abril de 2016). PM Modi's foreign policy: Making India a leading power. Hindustantimes. http://www.hindustantimes.com/analysis/pmmodi-s-foreign-policy-making-india-a-leading-power/story-SMXx2543j1uPgcHCb0QmJJ.html 
Nacht, P.A. (2013). El Dragón en América Latina: Las relaciones económico-comerciales y los riesgos para la región. Iconos. Revista de Ciencias Sociales, 45, 141-154. http://www.redalyc.org/articulo.oa?id=50925659010

Parameswaran, P. (21 de julio de 2015). A New 'Proactive' Indian Foreign Policy under Modi? The Diplomat. http://thediplomat.com/2015/07/ is-india-advancing-a-new-proactive-foreign-policy-under-modi/

Pérez Llana, C. (2013). Éxito económico, fracaso social. En Natanson, J. (Comp.), Explorador Le Monde diplomatique: India (pp. 23-26). Capital Intelectual.

Ragi, S. K., Sondhi, S., y Pathak, V. (2018). Introduction. En S.K. Ragi, S. Sondhi y V. Pathak (Eds.), Imagining India as a global power. Prospects and Challenges (pp. 6-21). Routledge

Rajan, R. S. y Sen, R. (2000). Trade Reforms In India Ten Years On: How Has It Fared Compared To Its East Asian Neighbours?". Center for International Economic Studies Discussion Paper (147). https://www.adelaide.edu.au/cies/ documents/papers/0147.pdf

Rao, N. (4 de marzo de 2016). New, strong and clear outreach. The Hindu. http://www.thehindu.com/opinion/op-ed/narendra-modi-new-strongand-clear-outreach/article8310200.ece?utm_source=InternalRef\&utm medium $=$ relatedNews\&utm_campaign $=$ RelatedNews

Rocha Valencia, A. y Morales Ruvalcaba, D.E. (2010). Potencias medias y regionales en el sistema político internacional: Dos modelos teóricos. Geopolítica(s). Revista de estudios sobre espacio y poder, 1(2), 251-279. https://revistas.ucm. es/index.php/GEOP/article/view/36329

Ross, C. (2010). India y América Latina y el Caribe: Relaciones económicas durante la Guerra Fría. The Asian Journal of Latin American Studies, 23(4), 7-42.

Sahni, V. (2011). Más estrategias que alianzas: Las nuevas relaciones internacionales de América Latina. En D. Wollard, G. Maihold y M. Mols (Eds.), La agenda internacional de América Latina: Entre nuevas y viejas alianzas (pp. 171-183). Nueva Sociedad.

Sahni, V. (2015). India and Latin America. Distant Acquaintance, Rhetorical Solidarity, Strategic Engagement. En S. Ganguly (Ed.), Engagening the world. India Foreign Policy since 1947 (pp. 375-398). Oxford University Press.

Seshasayee, H. (2020). The Ebb and Flow of India-Latin America Ties: Exploring Opportunities with Colombia. Observatorio América Latina Asia Pacifico, Documentos de Trabajo 002/20. https:/www.observatorioasiapacifico.org/ images/archivos/v-seminario/DT00220.pdf

Sharma, D. y Miklian, J. (2016). India's global foreign policy engagements - a new paradigm? Report. Norwegian Peacebuilding Resource Centre. https:// www.prio.org/Publications/Publication/?x=9125 
Siddiqui, H. (2015). América Latina tiene gran promesa para el comercio y la inversión de la India. Ministerio de Asuntos Exteriores. Gobierno de India. http:// mea.gov.in/in-focus-article-es.htm?25492/Latin+America+holds+huge+pro mise+for+Indian+trade+amp+investment

Soares de Lima, M. R. y Milani, C. (2016). Política Externa, Geopolítica e Modelos de Desenvolvimento. En M. R. Soares de Lima; C. Milani y E. Echart Muñoz (Eds.), Cooperación Sur-Sur, política exterior y modelos de desarrollo en América (pp. 21-40). CLACSO.

Tharoor, Sh. (2012). Pax Indica. India and the World of the Twenty-firt Century. Penguin Books.

United Nations Comtrade Database (UN Comtrade). (2019). https://comtrade. un.org/

Valles, M. (1999). Técnicas cualitativas de investigación social: Reflexión metodológica y práctica profesional. Síntesis S.A.

Vieytes, R. (2004). Metodología de la investigación en organizaciones, mercado y sociedad: Epistemología y técnicas. De las ciencias.

Viswanathan, R. (2008). Socios a largo plazo. Res Diplomática. Segunda época, $3,68-77$. 